Artículos

\title{
Agroecología y disputas sobre el desarrollo rural en Uruguay
}

Agroecology and disputes about rural development in Uruguay

Anabel Rieiro Castiñeira

Sistema Nacional de Investigadores, Agencia Nacional de

Investigación e Innovación. Facultad de Ciencias Sociales,

Universidad de la República, Uruguay

anabel.rieiro@cienciassociales.edu.uy

Gonzalo Karageuzián

Facultad de Ciencias Sociales, Universidad de la República,

Uruguay

gonzak419@gmail.com
DOI: https://doi.org/10.24215/15155994e147

Redalyc: http://www.redalyc.org/articulo.oa?id=84563515009

Recepción: 22 Agosto 2019

Aprobación: 23 Marzo 2020

\section{Resumen:}

El objetivo del presente trabajo será presentar información empírica sobre la historia y práctica actual de la Red de Agroecología del Uruguay, aportando interpretaciones posibles desde el enfoque sobre los antagonismos sociales rurales. Se trata de un estudio transversal no experimental, con triangulación de métodos (cualitativos y cuantitativos) y utilización de tres técnicas de recolección de datos: observación de plenarios, entrevistas colectivas y encuesta autoadministrada. Los resultados permiten concluir que, en los últimos años, fueron configurándose en Uruguay, tramas sociales locales de producción, distribución y consumo de alimentos agroecológicos, que, aun de forma precaria, sostienen prácticas y narrativas de resistencia al modelo hegemónico.

Palabras ClaVE: Agroecología, Desarrollo rural, Economía solidaria, Hegemonía, Uruguay.

\section{Abstract:}

The main objective will be to present empirical information about the history and current practices of the Uruguayan Agroecological Network, providing possible interpretations from the approach of rural social antagonisms. This is a nonexperimental cross-sectional case study, with triangulation of methods (qualitative and quantitative) and use of three data collection techniques: plenary observation, collective interviews and self-administered survey. The results allow us to conclude that, in recent years, local frames of production, distribution and consumption of agroecological foods were being set up in Uruguay, which, even in a precarious way, support practices and narratives of resistance to the hegemonic model.

KEYWORDS: Agroecology, Rural development, Solidarity economy, Hegemony, Uruguay.

\section{INTRODUCCIÓN}

La Red de Agroecología del Uruguay (RAU) es una articulación entre agricultores ecológicos, consumidores, procesadores, distribuidores de alimentos, diversas organizaciones sociales, instituciones y personas que promueven y desarrollan la agroecología porque ven en ella la capacidad de generar un impacto social y ambiental positivo.

El objetivo del artículo es presentar la sistematización de información empírica sobre la realidad histórica y las prácticas actuales de la RAU, aportando lecturas posibles sobre las luchas, los sentidos, el conflicto y la construcción de antagonismos en el contexto rural contemporáneo en Uruguay.

Nuestras preguntas-problemas fueron entonces las siguientes: ¿emergen desde estos entramados nuevas prácticas, sentidos, imaginarios sociales y condiciones de existencia en torno a los alimentos? ¿Cómo podrían interpretarse desde la discusión acerca de la construcción de hegemonía? 
Se parte de una noción epistemológica que retoma la agroecología como el estudio multidimensional de la estructura y función de los agroecosistemas, desde sus interrelaciones tanto ecológicas como culturales (Álvarez-Salas et al., 2014; León, 2012 y Altieri, 2010). En este sentido, describir algunas características generales y comprender subjetividades emergentes contemporáneas a partir del análisis de la RAU adquiere relevancia, en tanto acciones creativas y visiones alternativas -aquí y ahora- sobre el desarrollo rural, que a su vez nos permiten problematizar el modelo hegemónico actual en Uruguay, basado en el agronegocio y el extractivismo.

La pertinencia en partir de una experiencia concreta a través de un estudio de caso se fundamenta en la idea spinoziana de que "no sabemos lo que puede el cuerpo" (Deleuze, 2006, p. 27). Es decir, que son las propias prácticas y no solo las conciencias las que explican la construcción y composición del relacionamiento social actual en cada territorio. La composición de relaciones sociales y procesos asociativos como la RAU evidencian distintas luchas llevadas a cabo mediante dinámicas colectivas, desencadenadas a partir de la defensa de sistemas de producción agroecológicos, y de la producción familiar como forma de subsistencia.

Retomar estas experiencias es relevante, dado que permiten indagar sobre las disputas contemporáneas que se despliegan alrededor de los sistemas agroalimentarios, acercándonos a las relaciones sociales que allí se construyen, así como a las relaciones con la naturaleza, es decir, a los "modos de existencia" (Deleuze y Guattari, 2008, p. 200) asociados a ellos. Los modos de existencia dan cuenta de los propios procesos de subjetivación. Para Deleuze (2003), la posibilidad de transformación y creación social logra desencadenarse no cuando se persigue una apuesta utópica, sino cuando el acontecimiento deja emerger un nuevo sentido de lo posible. Un acto de creación en el que se organizan nuevos agenciamientos concretos, espacial y temporalmente, y que modifica sus condiciones de existencia.

El artículo está organizado en cuatro secciones. Primeramente, se explicitará el diseño, enfoque metodológico y técnicas aplicadas para la obtención de datos en el estudio de caso sobre la RAU. Seguidamente, se presentarán los antecedentes desde la sociología rural que permiten contextualizar histórica y territorialmente la actual construcción hegemónica nacional en cuanto a los modelos de producción agropecuaria. En la tercera sección se expondrán los resultados, organizados en tres apartados:

1. Historización de la RAU, presentando sus hitos principales y luchas llevadas a cabo por los entretejidos singulares que la conforman,

2. Perfil actual de la RAU y sus integrantes, su forma de organización, sus discursos y prácticas sociales,

3. Discusión sobre las interpretaciones posibles del caso en un contexto de disputa por modelos de desarrollo. En la cuarta y última sección del artículo dejaremos delineadas algunas conclusiones y reflexiones emergentes sobre cómo comprender la transformación social a partir del caso estudiado.

\section{Metodología}

El presente estudio de caso sobre la RAU es un estudio transversal no experimental, en el que se triangularon diferentes métodos (cualitativos y cuantitativos). Las tres técnicas de recolección de datos, que formaron parte de un plan de trabajo acordado entre la RAU y el Departamento de Sociología (DS) de la Facultad de Ciencias Sociales de la Universidad de la República (UdelaR), fueron:

1) Observación de los plenarios realizados por cada regional de la RAU entre mayo y julio del 2018. Se trató de una observación no-participante de seis de las siete regionales de la red, sobre los que se sistematizó la dinámica general, los temas tratados, el uso de la palabra, la forma de discutir, decidir y participar. Los plenarios contaron con una asistencia que varió entre 8 y 35 personas, y que duró aproximadamente un promedio de dos horas. 
2) Entrevistas colectivas a: la Coordinación Nacional, el grupo asesor y la asesoría técnica por un lado y a los coordinadores y representantes históricos de cada una de las siete regionales por otro. La selección de las personas que participaron en cada entrevista colectiva la realizaron los coordinadores de cada regional convocando a personas con un rol destacado o una importante trayectoria en la organización. Se realizaron siete entrevistas de aproximadamente una hora y media cada una a grupos que oscilaron entre 3 y 8 personas. Se elaboró una pauta semiestructurada, con nueve dimensiones generales que contenían cuatro preguntas guía cada una.

3) Encuesta autoadministrada a los 104 integrantes que asistieron a los plenarios de las regionales, realizados durante mayo, junio y julio del 2018. En todos los casos se tomó un tiempo antes del plenario para explicar el sentido y la lógica del cuestionario a ser completado por cada persona. Se trató de un formulario simple, que, además de recabar información sociodemográfica básica, contenía preguntas sobre las actividades específicas llevadas a cabo en el entorno de la RAU.

Los instrumentos y pautas confeccionadas para cada técnica se construyeron en diálogo con la coordinación nacional. Para el procesamiento y la sistematización de los datos se utilizaron los programas Atlas-Ti (entrevistas colectivas) y SPSS (encuesta). De esta manera, se analizaron las variables y relaciones más descriptivas desde la encuesta y se realizó un análisis del discurso de las entrevistas colectivas, contrastando las formas organizativas y discursos con las prácticas observadas durante los plenarios.

Los hallazgos principales fueron presentados oralmente en agosto del mismo año durante el V Encuentro Nacional de la RAU; allí, el objetivo fue recomponer el estado actual de la organización retomando una perspectiva dinámica e histórica, que le permitiera pensar sus limitantes y desafíos a futuro. Finalmente, fue presentado el informe escrito a la coordinación nacional en diciembre. Ambos espacios permitieron el intercambio y fueron retomados también como parte del trabajo de campo.

\section{ANTECEDENTES Y CONTEXTUALIZACIÓN: CONFORMACIÓN DEL MODELO RURAL HEGEMÓNICO}

El territorio sobre el cual hoy se sitúa la República Oriental del Uruguay fue de los últimos de América en ser colonizado por los españoles. La por entonces llamada Banda Oriental no contaba con minerales valiosos u otras riquezas, lo que postergó su efectiva exploración y expropiación por un par de siglos. Cuando esto sucedió, la ganadería introducida por los españoles se constituyó como la principal fuente de recursos. El ganado cimarrón era cazado para el uso del cuero y posteriormente para la carne salada o "charque", enviada a las colonias portuguesas y españolas como alimento de esclavos.

Los siglos XVIII y XIX vieron en la exportación de estos dos productos el sentido económico de la colonización española, mientras el sentido político era impedir que los portugueses se apropiaran del territorio. La tierra considerada "de poco valor" fue entregada en inmensas estancias por parte del rey de España a colonizadores o conquistadores como premio o pago por servicios a la Corona. Se consolidó así, al decir de Piñeiro (2014), la ganadería de grandes dimensiones como principal eje de la economía en la Banda Oriental; tanto, que luego de la independencia gran parte del territorio ya estaba otorgado en propiedad a partir de mecanismos legales.

En la segunda mitad del siglo XIX y tras importantes guerras civiles, desde sectores hacendados se impulsó un proceso de modernización de la campaña, que consistió en crear las condiciones para modificar la producción ganadera y dedicarse al creciente y demandante mercado inglés y europeo. Entre las medidas impulsadas se encontraba el alambramiento de los campos como forma de apropiarse definitivamente del territorio, y consolidar la propiedad de la tierra. Uruguay, que había sido el último territorio colonizado por los españoles, era ahora el primero en realizar esta delimitación. Otra medida que acompañó este proceso de modernización fue la introducción de razas inglesas de ganado para la producción de carne, y la de los ovinos, lo que rápidamente convirtió a la lana en la segunda fuente de riqueza. 
La regulación de las relaciones laborales en el campo dio lugar a la transformación del "gaucho" en peón rural, proceso que no estuvo exento de violencia por parte del Estado y de los estancieros, al decir de Piñeiro (2014). Lo cierto es que, como señalan Riella y Mascheroni (2019), la temprana asalarización de la fuerza de trabajo agrario no ha encontrado correlato en su consolidación como sector; históricamente ha sido uno de los grupos más vulnerables del país hasta el día de hoy.

El proyecto batllista y neobatllista signa la primera mitad del siglo XX, con una modernización basada en el Estado de Bienestar y la industrialización (Real de Azúa, 1984) llevada a cabo por una "hegemonía transformista" (Panizza, 1990). El conflicto entre el campo y la ciudad oculta y amortiza el disenso y la marginación de los sectores más pobres de cada contexto (Latorre, 1991; Real de Azúa, 1984).

El siglo XX fue así testigo de varios procesos en la sociedad rural uruguaya. Uno de ellos fue la creciente urbanización del país y el afianzamiento de una distribución profundamente desigual de la tierra (Piñeiro y Moraes, 2008). Como expone Piñeiro (2014), las explotaciones grandes (más de 1.000 hectáreas), a pesar de ser en gran parte del siglo en torno al $5 \%$ de las explotaciones totales, concentran alrededor del $55 \%$ de la tierra. Las producciones medianas (entre 100 y 999 hectáreas), con algunas fluctuaciones según los censos agropecuarios, más o menos son un $25 \%$ de las explotaciones, y concentran alrededor de un $35 \%$ de la tierra a lo largo del siglo. Por su parte, las explotaciones pequeñas (menos de 100 hectáreas) tuvieron un fuerte crecimiento en número hasta mitad de siglo y volvieron a decrecer hacia finales del mismo. A pesar de tratarse de la mayor proporción de explotaciones, en total nunca superan el $10 \%$ de la posesión de la tierra.

En este contexto, las asociaciones de pequeños productores familiares son uno de los actores relevantes en la defensa de la producción a pequeña escala, en la que una familia es propietaria de la tierra y utiliza mano de obra familiar, aunque eventualmente pueda contratar asalariados. Son predominantemente ganaderos, lo cual muestra que la matriz colonial perdura. De todas maneras, la producción familiar tuvo su auge a mediados de siglo en una etapa de industrialización, en la que también se dio un proceso de agriculturización del país, fundamentalmente a partir del impulso que se le dio a la producción de alimentos para abastecer el mercado interno, momento en que la pequeña producción creció en número, en tierras y en capacidad productiva. Como dice el autor citado a continuación:

La política de tierras en la historia de Uruguay puede ser mejor entendida si se tiene presente que siempre ha existido una tensión entre aquellos intereses políticos y económicos que veían a la tierra solo como un medio de producción y de apropiación de ganancias, contra una visión (fuertemente enraizada en el ideario artiguista) que sostenía que la tierra debía cumplir una función social, y debía ser utilizada para dar trabajo e ingresos a los que quisieran poblarla (Piñeiro, 2014, p. 219).

Estas dos miradas han logrado tener expresión en la sociedad a través de diferentes organizaciones sociales. En el campo uruguayo el número de organizaciones es cercano a 300, las cuales se diferencian por sus funciones, su cobertura (local, regional o nacional), la cantidad de integrantes, su representatividad, funcionamiento e incidencia política, así como por el logro de sus objetivos. Es evidente que no se puede presentar aquí ese número de organizaciones y la perspectiva histórica que cada una defiende respecto a la política de tierras en Uruguay, pero sí es importante tener presente que algunas de esas organizaciones han tenido y tienen un rol clave en los intentos de inclinar la balanza hacia un lado u otro.

Una de estas organizaciones es la Asociación Rural del Uruguay (ARU), fundada en 1871 en el contexto de modernización del campo, mencionado previamente. Se crea precisamente a impulso de los sectores ganaderos, que promovían dicha modernización, y tiene como objetivo la defensa y el fomento de los intereses de la producción agraria y de las industrias que la complementan y se derivan de ella. A lo largo de su historia ha jugado un papel de defensa de determinados sectores sociales ante el sistema político y el Estado, cumpliendo de esa manera funciones político-reivindicativas. Por su parte, la Federación Rural del Uruguay (FRU), surgida en 1915, por sus estatutos, su historia y accionar es el instrumento de representación y defensa de los grandes ganaderos (Fernández y Piñeiro, 2008).

Como expresión antagónica a estas dos organizaciones clásicas y asociadas a los sectores dominantes del campo, la defensa y representación de los intereses de los productores familiares y la pequeña producción se 
ha canalizado fundamentalmente a través de la Comisión Nacional de Fomento Rural (CNFR), compuesta por las sociedades de fomento rural y cooperativas rurales. Las sociedades de fomento rural aparecieron en el siglo XIX y su base social estaba compuesta por chacareros y agricultores con reclamos concretos respecto a caminería y fletes para comercializar su producción. La existencia de varias de estas organizaciones permitió en 1915 la creación de la Comisión Nacional de Fomento Rural. Su nacimiento supuso un esfuerzo por construir un instrumento gremial para los agricultores, ya que la ARU hasta ese momento solo representaba a los ganaderos. Además de estas, existen organizaciones de colonos, de mujeres, de asalariados, cooperativas, así como organizaciones de lecheros, arroceros, viticultores, etc. (Fernández y Piñeiro, 2008).

Con la entrada del siglo XXI, los procesos de urbanización y concentración de la tierra continuaron, pero ahora acompañados de otro fenómeno: la extranjerización. En el año 2000 el 90 \% de las tierras pertenecían a ciudadanos uruguayos, cifra que se redujo apenas una década después, hacia 2011, cuando pasa a ser solo el $54 \%$. Estas tierras perdidas por uruguayos las adquirieron personas jurídicas, principalmente sociedades anónimas, cuya nacionalidad se desconoce (Piñeiro y Cardeillac, 2018). Uno de los mecanismos que posibilitó dicho proceso fue una norma del año 1999, que derogó una disposición anterior que no permitía a las sociedades anónimas ser propietarias de tierras, por concebirlas como un bien común sujeto a la posibilidad de deterioro por malas prácticas de manejo. La eliminación de esta prohibición llevó a un aumento de la cantidad de compras por parte de estas sociedades.

En estas primeras décadas del siglo XXI se transformó radicalmente la producción agropecuaria en Uruguay, algunos de los procesos a destacar son el aumento exponencial de las operaciones de compra-venta de tierras, así como de los arrendamientos de estas, la mayor demanda internacional de energía y alimentos. En lo que refiere a la agricultura, hubo una expansión de las áreas productivas. El cultivo de soja estuvo al frente de dicho proceso, y se convirtió en el principal producto de exportación del país en el rubro agrícola. Según Riella y Romero (2014), su expansión tiene como base la introducción de paquetes tecnológicos, así como la utilización de variedades transgénicas.

Uruguay no fue ajeno a la expansión del agronegocio en América Latina. El sector de los granos mostró un aumento de su producción que casi triplicó al crecimiento del sector agropecuario en su totalidad, y es la soja el cultivo que mejor explica este crecimiento, dado que representa, para el año 2007 , el $30 \%$ del valor bruto de producción de la agricultura extensiva. De todas maneras, de las etapas del complejo agroindustrial sojero, Uruguay ha desarrollado principalmente la fase agrícola, la de acopio y la de exportación. En la fase preagrícola el país es dependiente de la importación de insumos y equipos para el desarrollo de los procesos productivos (Oyhantçabal y Narbondo, 2008). Entre 2005 y 2014 las importaciones de herbicidas en dólares aumentaron un $423 \%$; luego se observa un descenso sostenido hasta que en 2017 se llegaron a la mitad de lo que habían sido en 2014. En tanto, la importación de insecticidas aumentó un $577 \%$ entre 2005 y 2014 , y se observa una reducción en 2017 que representaba un gasto tres veces menor que el de 2014 (MGAP, 2017).

Respecto a la forestación, la misma se empieza a instaurar en el país en la década de 1960, dos décadas más tarde se encuentra un impulso vertiginoso de la forestación, a partir de decretos y leyes creados entre 1987 y 1990, que otorgan incentivos económicos e impositivos al sector. En paralelo, comienza la demanda de papel, respecto de la cual hay un proceso de deslocalización de la producción forestal. Las grandes empresas empiezan a producir en distintos lugares del mundo para asegurar la provisión de sus plantas, ubicadas en principio en el norte, con la celulosa producida en el sur. Luego se comienzan a construir centros industriales en los nuevos países productores. El primer gran impulso en cuanto a las plantaciones se da entre 1993 y 1996: se pasa de 200 ha a 42.000 ha anuales. Para el año 2000 había 661.000 ha plantadas, de las cuales 70 $\%$ se había realizado al amparo de la ley de 1987. Cuatro años más tarde había 1.402.144 forestadas -de las cuales $47 \%$ son plantaciones- y para 2011, 1.722.000 ha forestadas (Florit, 2011).

En Uruguay, hasta ese momento se habían instalado dos plantas procesadoras de celulosa: UPM, que en 2009 adquirió la mayor parte de las acciones de la empresa Metsä-Botnia, que había comenzado la construcción de la planta en 2005, y Montes del Plata, cuya planta, ubicada en el departamento de Colonia, 
comenzó a producir en 2014. En julio de 2019 UPM confirmó la instalación de otra planta procesadora de celulosa, lo cual ha sido motivo de conflictos vinculados a lo socioambiental. La cadena celulósica en Uruguay se encuentra consolidada y en crecimiento, y muestra de su peso económico en el país es que, al instalarse la tercera planta la celulosa, se posicionaría como primer rubro exportador (Boscana y Boragno, 2018).

En definitiva, como varios autores señalan (Cardeillac y Juncal, 2017; Riella et al., 2011), las relaciones capitalistas de producción han transformado el paisaje rural del Uruguay al instaurar un nuevo período en el que se ven especialmente afectadas la producción familiar y la producción a pequeña escala. ¿Cómo en este contexto lograron resistir los agricultores, consumidores, procesadores y distribuidores de alimentos agroecológicos?

\section{RESULTADOS Y DISCUSióN}

\section{Historias y narrativas de resistencias: la red de agroecología}

Paralelamente a la consolidación y el desarrollo del agronegocio como modelo hegemónico, encontramos a lo largo de estas décadas en el contexto nacional espacios que disputan dicha hegemonía. Emergen así colectivos como la Red Nacional de Semillas Criollas y Nativas del Uruguay, la Red de Agroecología del Uruguay, la Red de Grupos de Mujeres Rurales, la Asociación de Mujeres Rurales del Uruguay, entre otros, que contienen distintas narrativas sobre la ruralidad y cuestionan los valores y las prácticas normalizadas en este campo.

La materialidad alimentaria vive unida a un universo simbólico que guía la comprensión del mundo (Soler y Pérez, 2014), y en este sentido encontramos nuevas tramas sociales que parten de una visión crítica a la tendencia cada vez más industrializada y mercantilizada del sistema agroalimentario, tanto por sus efectos medioambientales, sus impactos en la salud humana, la amenaza a los sistemas de producción familiar y la polinización a través de las abejas, así como sus efectos en la pérdida de soberanía alimentaria y la creciente polarización y exclusión social.

[lo que caracteriza al modelo hegemónico actual es] la búsqueda de renta... o sea el lucro (...) y lo que apoya la agroecología, al revés, vamos a contracorriente, apoyamos lo familiar, lo ecológico, (...) te lleva a pensar tu propia vida, y ser coherente con eso no es changa (Productora, Regional Oeste - RAU).

Yo como cocinera (...) lo veo como un problema de salud, como cocinera, porque si comparo los nutrientes de un producto orgánico con uno no orgánico no es lo mismo (...) además, el segundo nos envenena. Después tenemos un problema medioambiental, tenemos contaminación de agua, contaminación de tierra, tenemos enfermedades asociadas a los productos agroquímicos (Procesadora, Regional Rocha - RAU)

Desde distintas motivaciones, concepciones y sentidos otorgados a la práctica, sea en la búsqueda económica por construir herramientas que permitan su sobrevivencia y mejora en la calidad de vida, o desde concepciones sociales, políticas y ecológicas inspiradas en la transformación social, varias personas construyen espacios de encuentro. La RAU es sin duda una de las redes más emblemáticas en el campo de la agroecología, de manera que resaltar algunos hitos emergentes de las historias que la atraviesan, así como sus luchas, es fundamental para comprender las relaciones sociales y sentidos construidos desde la organización.

Dados los escasos antecedentes encontrados al respecto (Gazzano y Gómez, 2015), se presentan a continuación algunos de los momentos resaltados como importantes por quienes integran la RAU, que aportan a la comprensión dinámica del fenómeno. En las entrevistas colectivas realizadas se repiten algunos momentos vivenciados como claves en la perspectiva de los participantes, tanto desde el proceso de la organización a nivel nacional como a nivel de sus regionales.

Si bien la práctica de la agroecología se remonta a los orígenes de la agricultura, aunque el uso contemporáneo del término data de los 70 (Hecht, 1999), las primeras experiencias en el campo de la agroecología se identifican por parte de los entrevistados tras la reapertura democrática en 1985. Durante 
el gobierno de Julio María Sanguinetti (1985-1990) se destacan al respecto dos contextos institucionales desde los que se comienza a trabajar en dicha perspectiva: las Organizaciones No Gubernamentales (ONGs Ambientalistas) y la Universidad de la República (UdelaR). En este último caso se identifica la Facultad de Agronomía como el espacio desde el cual se han ido desplegando progresivamente, desde hace más de 30 años, distintos proyectos, investigaciones, cursos a nivel de grado y posgrado (Gazzano y Gómez, 2015). En 1994, se realiza en Montevideo la primera feria de productos orgánicos, y un año más tarde comienzan a venderse dichos productos en los supermercados.

La Mesa de Agroecología del Uruguay es reconocida como antecedente importante de la agroecología en Uruguay. Estuvo conformada por el Centro Emmanuel, el Centro Uruguayo de Tecnologías Apropiadas (Ceuta), el Instituto de Promoción Económico Social del Uruguay (Ipru), Redes - Amigos de la Tierra, Foro Juvenil, el Grupo de Estudios sobre la Condición de la Mujer uruguaya (Grecmu), Restitución a la vida y Cáritas, con el objetivo de promover y difundir la agricultura de preservación y conservación de los recursos naturales y la salud humana. Algunas organizaciones de la Mesa de Agroecología integran desde 1992 el Movimiento Agroecológico Latinoamericano (Maela). Paralelamente, en el mismo año encontramos, por un lado, el primer decreto sobre certificación nacional, y, por otro lado, se conforma la Sociedad de Consumidores de Productos Biológicos (SCPB), gremial perteneciente a la ARU, en la que algunos productores orgánicos comienzan a juntarse.

Durante la segunda parte de la década de 1990 se encuentran los dos antecedentes organizacionales más directos de la RAU a través de la conformación de la Asociación de Productores Orgánicos del Uruguay (APODU) y la Asociación Certificadora de la Agricultura Ecológica-Uruguay (sello URUCERT). A mediados de 1996, a iniciativa de algunos productores y como respuesta a una disputa que se estaba dando entre las comercializadoras por los productos orgánicos, se convoca a productores, ONGs, comercializadoras, certificadoras y la Mesa de Agroecología a una reunión que dio en llamarse "El llamamiento de Crandon":

Había empezado a haber chisporroteos a nivel de los supermercados porque distintos productores chocaban a través de distintas comercializadoras. Empezó a haber problemas y para muchos era fundamental que los productores tuviéramos nuestra propia organización (entrevista colectiva, Coordinación Nacional)

En el año 2004 se identifican dos momentos importantes que explican un nuevo relacionamiento entre URUCERT y APODU, que da lugar a la RAU. En primer lugar, representantes de ambas organizaciones participaron de un Encuentro de "certificación alternativa" organizado por Maela e Ifoam. En el encuentro se planteaba, entre representantes de cinco continentes, la dificultad en el acceso a la certificación tercerizada o de tercera parte para los pequeños productores en Latinoamérica. Las dos organizaciones uruguayas habían acordado previamente constituir alternativas conjuntamente, aunque "Después se dejó de hablar de la certificación alternativa y se pasó a hablar de sistema de garantía participativa (...) Ese encuentro fue la previa al Foro Latinoamericano de garantías participativas en 2007" (entrevista colectiva, Coordinación Nacional).

El Sistema de Garantía Participativa (SGP) supone mayor accesibilidad a la certificación para pequeños y medianos productores, dada la reducción considerable del costo en comparación con el sistema de certificación tradicional (Vittori, 2018; Ifoam, 2008; Roldán et al., 2018). A la búsqueda de alternativas desde las organizaciones nacionales, avivada por el encuentro internacional, se le sumaron varios intercambios con la red brasilera Ecovida, a través de los cuales se comienzan a conocer formas concretas de certificación distintas a las de tercera parte.

En segundo lugar, un hecho señalado como clave para comprender la trayectoria organizacional de la red en dicho período es la propuesta a través de distintos decretos del gobierno sobre la certificación, a finales del 2004. La propuesta es interpretada por varios integrantes de APODU como restrictiva a empresas tradicionales, con impactos de exclusión para los pequeños productores familiares. Cuando esto se denuncia públicamente se abre un espacio de negociación con el fin de generar otro marco normativo. Las acciones de los integrantes de APODU y URUCERT dirigidas hacia la política pública sobre la certificación marcan 
una nueva etapa organizacional. Estas negociaciones se mantienen durante el gobierno de Tabaré Vázquez y se aprueba un nuevo marco normativo en el 2008.

Durante estos años de negociación varios hechos se van generando en el campo de la agroecología: la creación de la Red Nacional de Semillas Nativas y Criollas del Uruguay en 2004, la apertura de Ecotienda en un local de Ceuta, como resultado de la articulación entre APODU y el Grupo de Amigos y Consumidores de Productos Agroecológicos del Uruguay (GACPADU) en 2005. Finalmente, en mayo de 2005 se constituye la Red de Agroecología, y el mismo año se trabaja durante varios meses para constituir las regionales, conformándose la Regional Sur, la Regional Oeste, la Regional Maldonado y luego la Regional Treinta y Tres.

A partir de su constitución, la RAU ha realizado cinco encuentros nacionales. El primer encuentro nacional fue en noviembre de 2007, en vísperas del decreto gubernamental de 2008, por el cual se crea el Sistema Nacional de Certificación de la Producción Orgánica. El decreto reconoce dos maneras de certificación: entidades de certificación participativa y entidades de tercera parte, públicas y privadas. El reconocimiento de la certificación participativa es señalado como un logro, resultado de las acciones y negociaciones entabladas entre participantes de las organizaciones agroecológicas y algunos representantes del gobierno: "Con gran influencia de la Red, se reconoce el sistema de certificación participativa, cosa que en América Latina no era algo muy común (...) fue uno de los mayores logros de nuestra lucha” (entrevista colectiva, Regional Sur-Sur).

El segundo encuentro nacional se realizó en el 2009 en el departamento de Treinta y Tres junto a la Red Nacional de Semillas Nativas y Criollas. Durante el mismo año, a partir de distintos SGP que existían en la región, se crea el Foro Latinoamericano de Sistemas Participativos de Garantía. A nivel nacional, luego de haberse creado la Dirección General de Desarrollo Rural (DGDR) del Ministerio de Ganadería, Agricultura y Pesca en 2008, la RAU comienza a concretar distintos acuerdos y proyectos con dicha unidad. El primero se concretó en el 2011; se realizaron cursos de capacitación y se da comienzo a una etapa de consolidación institucional.

El tercer encuentro nacional se realiza en el 2012 en Nueva Helvecia. Se trató el tema de la agroecología y las políticas públicas; se invitó a varias personalidades nacionales e invitados de Argentina y Brasil. Fue el primer encuentro, que contó con financiamiento de la DGDR, abordó, además de las políticas públicas, el tema de la coordinación entre las distintas regionales. Dicha coordinación comenzó a funcionar a partir de este encuentro y en 2013 se aprobó el primer Proyecto de Fortalecimiento Institucional, a partir del cual "la organización comienza a fortalecerse, se le otorga mayor reconocimiento a la temática por parte del Estado y la sociedad en general. Es como que empezaron a dar más bolilla" (entrevista colectiva, Regional Oeste - RAU). Por su parte, con intención de favorecer la mayor territorialización, comienza a funcionar previo al encuentro la Regional Toronjil, como una parte de la Regional Sur que se autonomiza. Al año siguiente también se desprende de la misma la Regional Santoral.

En 2015 se organiza el cuarto encuentro nacional en Atlántida; se conforma también la Regional San José con énfasis en instalar la discusión sobre la importancia del rol de los consumidores en el desarrollo de la agroecología. La última regional en conformarse es la Regional Rocha en 2017. El quinto encuentro nacional se realiza del 10 al 12 de agosto de 2018 en Canelones. Por primera vez se realiza un encuentro nacional entre mujeres de la agroecología, que indaga en la historia desde la perspectiva de las mujeres.

A trece años de su creación, la RAU se ha ido fortaleciendo organizativamente; cuenta hoy con siete proyectos en marcha, de los cuales cuatro emergen de alguna de sus regionales y otros tres se ejecutan a nivel nacional. En cuanto a estos tres últimos, encontramos un proyecto de fortalecimiento institucional y agricultura, que surge a nivel nacional, y el Proyecto de Ovinos, que emerge desde la Regional Toronjil, para ampliarse luego a toda la RAU. Las distintas trayectorias construidas a nivel local, regional y nacional han ido configurando una estructura descentralizada, que conjuga particularidades de los colectivos a nivel regional, coordinaciones nacionales basadas en la representación y encuentros nacionales. 


\section{Nuevas tramas agroecológicas en el Uruguay contemporáneo}

La RAU, junto a la Red Nacional de Semillas Nativas y Criollas y a la Sociedad Científica Latinoamericana de Agroecología definen a la agroecología como una ciencia que "integra y aplica el conocimiento tradicional y las prácticas culturales de los agricultores y las agriculturas, para diseñar y manejar agroecosistemas productivos, diversificados y resilientes, con el objetivo de alcanzar la soberanía alimentaria y la justicia social" (Charbonier, Malán y Bassani, 2017, p. 25). La RAU rige su vida institucional según diversos espacios de encuentro, que toman el formato de asambleas, comités, espacios de formación, comisiones, coordinaciones y plenarios; desde ellos se configuran relaciones e intercambios sociales en el ámbito local, nacional y regional, con el objetivo de fortalecer las prácticas agroecológicas.

Como muestra el siguiente cuadro, los espacios más importantes de la RAU desde la organización social son: los plenarios nacionales (instancias bianuales abiertas a todos/as los/as integrantes de la RAU), las siete regionales territoriales (con plenarios bimensuales) -cada una de las cuales cuenta con su comité de ética y calidad, sus coordinadores y en la mayoría de los casos una secretaría técnica-, la Coordinación Nacional (con reuniones bimensuales alternadas a los plenarios regionales) -integrada por dos coordinadores de cada regional y la secretaría técnica-, y, por último, se cuenta con dos espacios de asesoramiento mayormente vinculados a la certificación: el grupo asesor y la secretaría técnica general.

FIGURA 1

Estructura organizativa para el funcionamiento general de la RAU

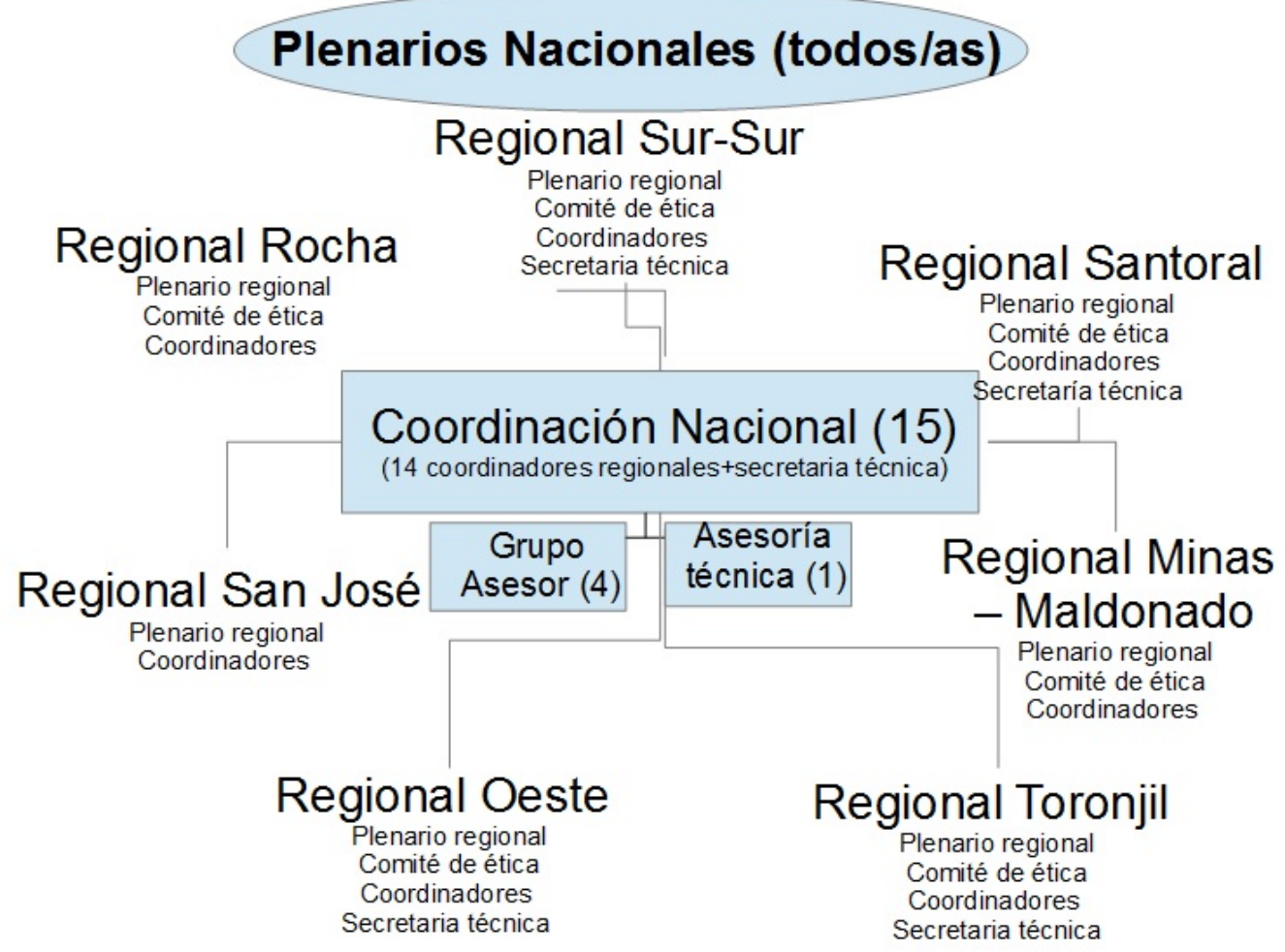

Elaboración propia con base en entrevistas colectivas

Bajo una estructura horizontal, rizomática y descentralizada, la RAU define sus prioridades y estrategias generales en los plenarios bianuales, y organiza su vida cotidiana a partir del trabajo de las regionales, coordinaciones nacionales, etc. Es decir, sus integrantes construyen espacios compartidos para poner en común los problemas vinculados a las prácticas agroecológicas, y buscan soluciones colectivas. 
Actualmente la RAU está conformada aproximadamente por 213 núcleos territoriales, representados en el 53,5\% de los casos por mujeres y en el $46,5 \%$ por hombres. Los núcleos territoriales son las personas que participan directamente en la red, quienes en algunos casos representan individuos y en otros a sus grupos familiares y/o sus colectivos de producción y/o consumo. Agrupados en torno a siete regionales de la zona sur del país, como muestra la tabla a continuación, cada regional presenta características específicas, lo que delinea una red heterogénea, tanto en el número de integrantes, como en su trayectoria, su perfil productivo y sociodemográfico.

TABLA 1

Información general de las siete Regionales: inicio de funcionamiento, edad media, porcentaje de mujeres y hombres, cantidad de integrantes

\begin{tabular}{|l|l|l|l|l|l|}
\hline Regional & $\begin{array}{l}\text { Inicio de } \\
\text { la regional }\end{array}$ & $\begin{array}{l}\text { Edad media } \\
\text { de sus } \\
\text { integrantes }\end{array}$ & \% Mujeres & \% Hombres & $\begin{array}{l}\text { Cantidad } \\
\text { actual de } \\
\text { integrantes }\end{array}$ \\
\hline Santoral & 2011 & 46 & 12,9 & 87,1 & 31 \\
\hline Oeste & 2005 & 48 & 52,2 & 47,8 & 23 \\
\hline San José & 2015 & 47 & 70 & 30 & 40 \\
\hline Sur-Sur & 2005 & 54 & 48,4 & 51,6 & 31 \\
\hline Toronjil & $(2005)$ & 46 & 70 & 30 & 50 \\
\hline Rocha & 2011 & 36 & 52,4 & 47,6 & 21 \\
\hline Minas & 2008 & 53 & 52,9 & 47,1 & 17 \\
\hline Total & & & & 213 \\
\hline
\end{tabular}

Elaboración propia con base en entrevistas colectivas y encuesta, 2018

Las particularidades de cada regional no solo refieren a actividades y número de integrantes (notemos que hay regionales como Toronjil con 50 integrantes, y Minas con 17 integrantes), sino también a su año de constitución y rangos etarios de las personas que las conforman. En este sentido, la edad media de la RAU es de 47 años, similar a la edad promedio de los pequeños productores familiares en Uruguay, que, según el Ministerio de Ganadería Agricultura y Pesca (MGAP) (2014), es de 50 años. Sin embargo, existen diferencias etarias entre las regionales, y el contraste puede ejemplificarse entre la Regional Sur-Sur y la Regional Rocha, en las que se cuenta con un promedio de edad de 54 y 36 años respectivamente.

En cuanto a la conformación de colectivos, instituciones y organizaciones dentro de las distintas regionales, se relevaron a nivel asociativo: tres colectivos de producción en la Regional Santoral (Santa Rosa, Punto Verde y Calpamás), un colectivo de mujeres productoras en la Regional Toronjil (Calmañana), dos colectivos de consumo en la Regional Sur-Sur (Copau y Asobaco) y recientemente vinculados a Rocha y Minas un colectivo de colonos y el Grupo Minas respectivamente. En lo referente a instituciones públicas (que integran la regional con voz pero no voto), únicamente se relevó al Centro Ecológico Integrado al Medio Rural (CEIMER) en Rocha. Por último, a nivel de ONGs ambientalistas encontramos al Centro Uruguayo de Tecnologías Apropiadas (CEUTA) como parte integrante de la RAU.

En el $66 \%$ de los casos se trata de núcleos que se integran a la RAU definiendo a la producción como su actividad principal. Sin embargo, de la información sistematizada mediante la encuesta realizada a 104 participantes se desprende que, más allá de sus tareas principales, se adoptan en la mayoría de los 
casos esquemas de pluriactividad. Es decir, varios integrantes además de producir productos orgánicos, los procesan, comercializan, consumen, así como asumen tareas de gestión o trabajan como técnicos.

TABLA 2

Tipo de actividad principal del núcleo, según regional

\begin{tabular}{|l|l|l|l|l|l|l|l|}
\hline & Producción & Comercio & Consumo & Procesado & Gestión & Técnico & Total \\
\hline Santoral & 26 & 0 & 0 & 0 & 1 & 4 & 31 \\
\hline Oeste & 15 & 0 & 5 & 0 & 1 & 2 & 23 \\
\hline $\begin{array}{l}\text { San } \\
\text { José }\end{array}$ & 5 & 0 & 20 & 0 & 0 & 15 & 40 \\
\hline Sur & 23 & 0 & 6 & 1 & 1 & 0 & 31 \\
\hline Toronjil & 44 & 3 & 2 & 1 & 1 & 0 & 51 \\
\hline Rocha & 11 & 0 & 7 & 2 & 0 & 0 & 20 \\
\hline Minas & 17 & 0 & 0 & 0 & 0 & 0 & 17 \\
\hline Total & 141 & 3 & 40 & 4 & 4 & 21 & 213 \\
\hline
\end{tabular}

Elaboración propia con base en entrevistas colectivas

En cuanto a los/as productores/as se trata casi en su totalidad de prácticas de producción familiar a pequeña escala. Según la encuesta realizada, en el $80 \%$ de los casos se poseen menos de 10 hectáreas utilizadas de manera agroecológica, con predominancia de la producción de hortalizas (aunque también se producen frutas, quesos, plantas, etc.). Además son en su mayoría (55\%) propietarios de la tierra en la que producen, lo que permite una relación con los medios de producción particular, distinta a la que se establece cuando se arriendan amplias extensiones. El total de tierra destinada a la producción agroecológica desde la RAU se estima, según la encuesta a productores, en 1.000 hectáreas.

Conviven en el grupo de productores quienes han sido tradicionalmente productores rurales, con algunos recientemente emigrados del medio urbano. Una hipótesis derivada de algunos discursos emergentes durante el trabajo de campo es que los productores rurales encuentran en la producción orgánica una herramienta para poder continuar produciendo a escala familiar, mientras que los que emigran del medio urbano buscan mayormente una alternativa al modo de vida citadino y la concreción de ideales ecológicos. Sin duda, los sistemas culturales y entornos diferentes de los que provienen sus integrantes inciden en la multiplicidad de sentidos, saberes y motivaciones que atraviesan a la red.

La vida cotidiana de la organización gira en torno a temas vinculados a la agroecología, entre los cuales predomina el tema de la "certificación" de la producción orgánica, que se realiza formal y jurídicamente a través de la Asociación ACAEU, certificadora que, además de las certificaciones agropecuarias otorgadas a integrantes de la RAU por el Sistema de Garantía Participativo (SGP), certifica a empresas y productos de empresas productoras e importadoras.

Los productores necesitan el sello para poder vender mejor (...) eso no garantiza una concepción integral sobre la agroecología, pero los grupos tienen historia y se van integrando (...) y van viendo que este sistema es más que lo orgánico (Entrevista colectiva, Regional Sur-Sur - RAU).

Nosotros ahora estamos viendo que, además de la certificación, deberíamos tomar postura, discutir sobre montones de cosas... tendríamos que participar más. Estamos viendo cómo no pertenecer a una regional solo por la certificación, sino incorporando todos los temas que hacen a la agroecología (Entrevista colectiva, Regional Santoral - RAU) 
La certificación a través del Sistema de Garantía Participativa (SGP) supone un tiempo de dedicación alto en la vida institucional, pero también garantiza aspectos claves para su sostenibilidad. Según los datos de la encuesta, aproximadamente el $67 \%$ de los productores de la RAU están certificados, el 11\% está tramitando la certificación y el 22\% no lo está. La certificación participativa es una conquista política de la organización para garantizar el acceso/derecho de los pequeños productores al sello que distingue la agricultura orgánica de la agricultura tradicional.

Sin embargo, como todo proceso de regulación, la certificación conlleva varias gestiones burocráticas que resolver, en este caso colectivamente. El productor que quiera certificar su producción deberá llenar un formulario detallando el manejo y uso de su terreno, pagar un arancel y recibir al comité de ética y calidad en su predio. En general la secretaría técnica regional (en conexión con el grupo asesor y la asesoría técnica nacional) coordina junto al comité de ética local (conformado por productores, consumidores y técnicos) una visita al lugar de la producción, durante la cual se registra, verifica y certifica el uso y manejo agroecológico de la producción.

La certificación supone el reconocimiento diferencial de productos orgánicos, lo que a su vez mejora las vías de comercialización. Este objetivo en algunos casos individuales pasa a ser la motivación principal para la participación. Encontramos así, al igual que en algunos grupos agroecológicos en Florianópolis (Warmling y Moretti-Pires, 2017), que los sentidos atribuidos a la agroecología por los propios integrantes de la RAU se articulan en dos ejes: 1) las cuestiones productivas y la inserción al mercado (en donde encontramos como herramienta la certificación), y 2) la promoción de la agroecología como construcción de otro modelo socioproductivo. Así, una de las preocupaciones más frecuentes pronunciadas por los coordinadores nacionales son las limitantes que encuentra la RAU para promover la agroecología en su concepción amplia, integral y holística, frente a una dinámica más rutinizada, que se instala para poder cumplir con los procesos de certificación. De esta manera, se encuentra que aunque estos sentidos pueden ser complementarios, por momentos la inserción en los mercados y la construcción de otro modelo integral de sociedad se plantean de forma contradictoria, hecho que se transforma en una de las principales tensiones organizacionales.

En cuanto a las prácticas sociales cotidianas de la organización, según las entrevistas colectivas, el mecanismo mediante el cual se toman las decisiones suele ser el consenso, para lo cual se valora el intercambio y la discusión. No obstante, en algunos temas en los que no se llega al consenso, se utiliza el voto y se decide por mayoría.

La toma de decisiones por consenso no marca dinámicas colectivas homogéneas en referencia a la participación y al uso de la palabra. En este sentido, en algunas regionales hay liderazgos marcados que tienen un peso importante en las discusiones y las orientaciones generales, mientras que en otras existe una dinámica horizontal, rotativa, sin referentes claros. No obstante, en todas las regionales hay una importante circulación de la palabra en los momentos de discusión colectiva. Esto pudo corroborarse durante la observación que se realizó en seis de los siete plenarios regionales, aunque con algunas diferencias en los niveles de circulación de la misma.

Respecto a la participación, emerge como un problema en las entrevistas realizadas la falta de motivación y de asistencia a los plenarios por parte de algunas personas. Varias regionales han tomado incluso medidas para abordar el problema, exigiendo un porcentaje mínimo de asistencias a los plenarios para otorgar la certificación. De la asistencia observada durante los plenarios entre mayo y julio del 2018, puede decirse que más de la mitad de los integrantes concurrieron a la convocatoria territorial. En relación a la autopercepción de dichos participantes acerca de su grado de involucramiento con la organización, de 96 personas que contestaron la pregunta de la encuesta, el $85 \%$ señala entre medio, alto y muy alto su grado de involucramiento con la organización. 


\section{Interpretaciones posibles en un contexto de antagonismos y disputas sobre los modelos de desarrollo rural}

Para abordar la cuestión de la hegemonía se retoman las prácticas sociales que atraviesan la cotidianeidad de las personas y la vida colectiva, con el fin de analizar los sentidos atribuidos a la agroecología por los propios actores de la RAU. Más allá de "la política" entendida como el campo en el que se desarrollan las prácticas institucionales de la política convencional, es interesante observar "lo político" como modos en los que se instituyen las sociedades a través de experiencias singulares y praxis de autoconstitución en el campo de la acción colectiva y las organizaciones sociales.

En el campo uruguayo, la dinámica de posiciones (y disposiciones) que estructuran y organizan los sistemas de significación y las prácticas acordes ha estado basada históricamente en el conflicto "ciudad/campo", un antagonismo hegemónico que pocas veces ha dejado grietas desde donde pudieran emerger disputas que refieren al tipo de desarrollo rural que se pretende impulsar. Cuando estas aparecen, a lo sumo, se asocian al conflicto entre grandes productores y pequeños productores, pero no al modo de producción adoptado por ellos más allá de la escala.

La urbanización y el proceso de modernización/democratización (Panizza, 1990) instaurado a principios del siglo pasado, basado en cánones occidentales, conforman una sociedad particular en su vinculación con la producción rural y el desarrollo, ambos asociados fuertemente con el crecimiento económico. Como se mostró anteriormente, podemos presenciar cómo en las últimas décadas se configura hegemónicamente en Uruguay la asociación entre modernización del campo y el agronegocio, lo cual frecuentemente termina asociando los otros tipos de producción, como la orgánica, a la producción tradicional, sinónimo de atraso e ineficiencia.

Si se retoma el tema de la hegemonía -entendida como construcción política donde toda particularidad asume un rol universal, subyacente a través de vínculos equivalenciales entre demandas heterogéneas entre las que se reconocen dentro de un mismo campo antagónico (Laclau, 2008; Laclau y Mouffe, 2010)-, podríamos decir que, desde los distintos territorios que conforman la RAU, existe un sentido compartido sobre la agroecología como modelo antagónico al modelo hegemónico actual, el cual es entendido en ocasiones como "un modelo agroexportador, agroindustrial, que apunta a lo productivo entendido solo como ganancia. (...) pero bueno, se puede producir sin echar químicos, sin contaminar. Eso es así... nosotros lo hacemos" (Entrevista colectiva, Regional Santoral - RAU).

El agronegocio es industrial (...) yo tengo un tractor chico y hacerle tomar conciencia a la gente, al consumidor de frutas orgánicas, por lo menos en Rocha ciudad ha costado muchísimo (...) las empresas te siguen ganando y a la gente le da lo mismo, te venden una verdura artificial preciosa o natural con picaduras (...) se van y compran la que está $100 \%$ preciosa; tenés que explicar, y eso es más trabajo (Productor, Regional Rocha- RAU).

Los discursos emergentes de las entrevistas colectivas realizadas en las distintas regionales configuran visiones compartidas sobre ciertos temas y disensos sobre otros. Por un lado, encontramos ciertas nociones y sentidos comunes otorgados a la agroecología en cuanto a la salud y sustentabilidad medioambiental, entendidas como modelo ecológicamente alternativo y superior (no atrasado) respecto al modelo de producción convencional. Este modelo es caracterizado según los integrantes de la red por requerir una alta inversión de capital, utilizar mayor tecnificación, usar agrotóxicos y plaguicidas y hacer uso intensivo del suelo a gran escala.

También encontramos en los distintos discursos que emergen, algunos actores reivindicados como “aliados”. Entre ellos los dos más mencionados son: la Red Nacional de Semillas Nativas y Criollas (se señala gran coincidencia en su enfoque agroecológico) y las sociedades de fomento rural (cercanas en cuanto a la defensa de la producción familiar). Sin embargo, hay grandes divergencias a la hora de construir una lectura compartida sobre los aliados y adversarios de la RAU. Por ejemplo, si bien suele existir de manera generalizada 
cierto alejamiento de las gremiales que representan los intereses de los grandes empresarios en el campo, emergen discursos casi opuestos frente a los "autoconvocados" - un actor colectivo de composición difusa que emergió recientemente reclamando mayor rentabilidad del "campo"-, por los cuales se argumenta que los gastos en tarifas, combustible, e impuestos son elevados: "Tuvimos en nuestra regional un problema, porque un participante fue a una manifestación de los autoconvocados y planteó que la regional debería tener presencia en ese espacio" (Entrevista colectiva, Regional Sur-Sur - RAU)

[los autoconvocados] Están muy enojados por el tema del dinero, pero son los que más se han beneficiado del campo en estos años (...) no se quejan ni un segundo de todo lo que se ha perdido de suelo, de toda la contaminación del agua. No existe, dentro de su discurso ¡No existe!, tampoco de pequeños y medianos productores se habla nada (...) No hay una propuesta de cambiar el modelo productivo (Entrevista colectiva, Regional San José - RAU).

$\mathrm{Al}$ preguntar por los "aliados", más allá de las asociaciones rurales de pequeños productores y las asociaciones que defienden los principios agroecológicos, se nota una ausencia casi completa de referencias a actores sociales históricos en Uruguay, como la central sindical, la Federación Uruguaya de Cooperativas de Vivienda por Ayuda Mutua (Fucvam), etc. En este sentido, no existe como organización un relacionamiento con el campo popular, ni con movimientos urbanos en los que las demandas de otros actores sean apropiadas por la RAU generando equivalencias.

Frente al Estado, tampoco parece haber consenso, algunos participantes sienten demasiada cercanía de la RAU con actores gubernamentales, hecho que interpretan negativamente por restar fuerza a la organización y su poder de presión. Otros participantes sienten al Estado como un espacio clave a disputar y un actor aliado para lograr algunas políticas públicas favorables para el sector.

Tengo una discrepancia con la red, creo que está muy volcada a los actores gubernamentales y eso es un proceso hasta contradictorio y negativo, porque se trabaja el Plan y eso es a través de una ley y la ley lo hacen los parlamentarios (...) y para mi hay un error (...) se apunta mucho a los actores gubernamentales y se pierde fuerza con eso de que tengo que negociar y bajar el tono.

Yo creo que esos no son socios-aliados, sino donde vos tenes que ir a presionar para que se aprueben las leyes y eso. Los verdaderos socios son (...) la Red de Semillas, el Socla, y se fueron sumando algunas facultades... esos son los socios, los otros son a los que vos le vas a demandar (...) yo creo que las dos cosas van juntas, para conseguir presionar necesitas movilización, no es una u otra (Entrevista colectiva, Regional Sur-Sur - RAU).

Los discursos, retomados como organizadores de los sistemas de significación, plantean así una heterogeneidad de visiones que conviven en la organización. Existen también distintos grados de politización en los distintos territorios, lo que se convierte en un problema a la hora de querer emitir comunicados sobre la coyuntura actual, y se traduce organizacionalmente en tensiones entre modelos de gestión más centralizados o descentralizados. Algunos participantes sienten que, además de las propias prácticas agroecológicas, la RAU debería expresarse con mayor frecuencia públicamente ante distintas circunstancias, mientras otros participantes sostienen que no debería posicionarse frente a los temas en los que aún no existe una postura compartida.

¿Debería interpretarse esta característica como sinónimo de despolitización o incapacidad para formar parte de relaciones antagónicas y modos de significación alternativos? Se cree necesario hacer un desplazamiento a la propia pregunta, para analizar la construcción de la hegemonía, no solo desde los modos de significación, sino también desde los modos de existencia.

Como plantea Gutiérrez (2017), el capital no tiene un adentro y un afuera, sino que avanza según una topografía distinta, a través de mallas de relaciones sociales que habilitan la maximización de ganancia de algunos y el empobrecimiento de otros. Es en este sentido que se observa que las distintas tramas sociales conformadas en torno a la RAU, si bien no se plantean ni ponen en crisis el capital, cultivan capacidades asociativas diferentes a lo que este produce, generando asociaciones que permiten resistir a una única manera de producir, distribuir y consumir alimentos. 
Las experiencias situadas y concretas, en donde las personas, familias y colectivos deciden producir agroecológicamente, proponen distintos modos de gestionar la naturaleza, alejándose de la lógica predominante, que la concibe solo como fuente de riqueza, y conformando relaciones sociales sobre la base de los procesos naturales. Desde estas cotidianidades y prácticas concretas se posibilitan ciertos hilos reconocibles y compartibles entre productores, consumidores, procesadores, distribuidores y técnicos, que retoman la naturaleza no solo como medio de producción con el cual enriquecerse, sino tambén como medio indispensable para la existencia actual y futura de las personas.

El desafío se plantea entonces en cómo repensar la transformación a partir del eje capital/vida (PérezOrozco, 2014; Gago, 2014; Gutiérrez, 2017), haciendo posibles nuevos procesos de politización y subjetivación. La RAU, a partir del reconocimiento su heterogénea composición, logra llevar adelante, por un lado, algunas prácticas cotidianas como la producción orgánica, la distribución directa y el consumo responsable, y, por otro lado, encarna acciones en común como la lucha por el reconocimiento de la certificación participativa, la elaboración de varios comunicados a la opinión pública (por ejemplo contra la Ley de Riego) y el impulso junto a otras organizaciones del Plan Nacional de Agroecología (PNA).

Sin duda, dicho Plan marca el contexto actual y condicionará el despliegue o repliegue de la producción agroecológica a mayor escala. Es seguramente en esta ley donde se hace mayormente explícita la disputa con el modelo hegemónico. A modo de ejemplo, cuando se juntaron firmas para impulsar el plan, la carta dirigida al anterior presidente decía: "Consideramos que asistimos a un proceso de intensificación agropecuario, impulsado por el crecimiento económico de la producción exportable, que presenta impactos sobre la sustentabilidad ambiental, social y económica”. A partir de allí, se propone la agroecología como estrategia alternativa, y como objetivos principales del PNA, promover la soberanía alimentaria, facilitar procesos de transición a la agroecología, promover el acceso a la tierra para pequeños productores, la participación en el territorio.

La agroecología, entendida como un sistema (Altieri, 1999), frecuentemente se piensa y teoriza como un tipo ideal "fuera del capitalismo", un modelo anticapitalista que difícilmente se encuentre en estado puro en la realidad. Tomemos como ejemplo la comercialización: existen en la RAU declaraciones y orientaciones generales que promueven la venta directa entre productores y consumidores, el comercio local a través de ferias por ejemplo, y el fortalecimiento de los grupos de consumidores. Sin embargo, más allá de las diversas experiencias de relaciones más horizontales y humanas entre producción y consumo, estas conviven con formas de comercialización típicas del capitalismo, entre las que el caso más claro es el de la venta en supermercados de diverso tamaño y alcance.

Quizás las grandes superficies sean según los principios, las menos deseadas (...) La orientación es a fortalecer la cercanía y venta lo más directa posible, pero no podés ir con toda la teoría cuando sabes que en la práctica no hay una situación que permita tener los niveles de comercialización que querríamos todos (Entrevista colectiva, Regional Toronjil - RAU).

Si bien esto evidencia ciertas tensiones en cuanto a los canales de comercialización, la propia contradicción señala un horizonte hacia construir espacios alternativos a las formas hegemónicas de comercializar, en el entendido de que es una instancia privilegiada para construir cotidianamente desde lo relacional a partir de los espacios de encuentro que se van generando.

\section{CONCLUSIONES: LA AGROECOLOGÍA Y LA TRANSFORMACIÓN SOCIAL}

Tras haber presentado la información empírica y el análisis de los discursos de los participantes de la RAU, podemos retornar a la pregunta-problema planteada al comienzo del artículo sobre la posible interpretación de los nuevos entramados agroecológicos configurados en torno a los alimentos, desde el punto de vista de la construcción de antagonismos y hegemonía en el contexto rural uruguayo. 
Una primera reflexión la amerita el hecho de que sea en un contexto neodesarrollista (con líneas de continuidad con el proyecto neoliberal en cuanto al modelo de acumulación) en el que emerja la RAU. Paralelamente a la expansión del agronegocio como paradigma en la producción agropecuaria, nace una red que defiende la producción familiar, desde una concepción agroecológica. En este sentido, la disputa puede interpretarse desde los históricos disensos sobre el uso de la tierra en Uruguay, siendo la RAU una expresión de las que sostiene que la tierra tiene un fin socioambiental, y no es solamente un medio para producir y acumular capital. Sus demandas y luchas emergentes, como la defensa de la certificación participativa, han permitido en esta década y media de existencia ir generando sentidos compartidos respecto a una pluralidad de prácticas de producción, distribución y consumo de alimentos. La historicidad de la organización demuestra entonces cómo se van renovando y consolidando nuevas demandas, reivindicaciones e imaginarios posibles sobre los alimentos desde las prácticas y luchas desplegadas.

La RAU complementa prácticas productivas y reproductivas alternativas al modelo convencional de producción, defendiendo en común la habilitación, difusión y defensa de la agroecología. Estas prácticas van desde lo individual, lo grupal y lo colectivo, conjugando modos de producir, procesos deliberativos y modelos de representación, que dan cuenta de una organización compleja y heterogénea. Compuesta predominantemente por pequeños productores familiares propietarios, mantiene vínculos estrechos con organizaciones que defienden la producción de los pequeños productores, como la Comisión Nacional de Fomento Rural, pero incorpora además de la escala, la dimensión ambiental. En este sentido, se articula con otras organizaciones que defienden la agroecología como la Red de Semillas Criollas y Nativas, grupos de académicos y universitarios afines, las Organizaciones No Gubernamentales ambientalistas, etc.

Se puede concluir entonces que, más allá de las propias contradicciones constitutivas de la red, sus dinámicas asociativas ponen en evidencia el antagonismo socioambiental en los modos y modelos de producción, distribución y consumo de los alimentos. Un antagonismo invisibilizado, ausente o negado bajo el consenso neoliberal que impone la racionalidad instrumental como lógica única, hegemónica a la hora de explicar "la" economía. En consonancia con otras investigaciones (Duché-García et al., 2017; Serra Borsatto y Simoes do Carmo, 2012), podemos decir que los entramados colectivos en defensa de las prácticas agroecológicas en Uruguay cuestionan la adopción de principios "universales" de racionalidad económica deslindada de la forma de generar sociedad y su vinculación con la naturaleza. Encuentran así modos de resistir a la tendencia hegemónica que caracteriza a la dinámica del capital oligopólico.

Por último, y como reflexión final, mucho tiempo hemos construido desde las ciencias sociales marcos teóricos de interpretación sobre la transformación social basados en categorías duales, retomadas como contradicciones ideales y explicativas, con capacidad para develar la realidad de los sistemas comprendidos como un todo coherente. Sin embargo, estas perspectivas no logran dar cuenta de la dinámica compleja, racional y afectiva que se plasma a nivel territorial, en donde coexisten una multiplicidad de oposiciones reales, que se entrelazan e imbrican en las relaciones sociales y estructuras de poder. El desafío de esta investigación fue, entonces, comprender cómo a partir de la pluralidad de las experiencias concretas y prácticas específicas agroecológicas que contienen sentidos y significados diversos en torno a la alimentación, mediante la conformación de redes, se construyen relaciones sociales entre los participantes, quienes encuentran formas de autoorganizarse a partir de intereses y necesidades en común. En este sentido, desde su irreductible heterogeneidad territorial y como resultado de múltiples contradicciones y conflictos pueden captarse en la RAU componentes asociativos y performativos no convencionales, en su carácter de resiliencia y potencia hacia la transformación social, sin tener que ser reducidos a lecturas que homogenicen e interpreten dichas capacidades desde una racionalidad única o un antagonismo dual. 


\section{REFERENCIAS}

Altieri, M. (1999). Agroecologia: Bases cientificas para una agricultura sustentable. Montevideo: Ed. NordanComunidad.

Álvarez-Salas, L., Polanco-Echeverry, D., y Ríos-Osorio, L. (2014). Reflexiones acerca de los aspectos epistemológicos de la agroecología. Cuadernos de Desarrollo Rural, 11(74), 55-74. Recuperado de https://revistas.javeriana.edu. co/index.php/desarrolloRural//issue/view/711

Boscana, M., y Boragno, L. (2018). Actualidad del sector forestal. En Anuario OPYPA (pp.229-239). Montevideo: Ministerio de Ganadería, Agricultura y Pesca. Recuperado de http://www.mgap.gub.uy/unidad-organizativa/o ficina-de-programacion-y-politicas-agropecuarias/publicaciones/anuarios-opypa/2018

Cardeillac Gulla, J., y Juncal Pérez, A. (2017). Estructura agraria y trabajo en un contexto de cambios: el caso de Uruguay. Mundo Agrario, 18(39), e072. https://doi.org/10.24215/15155994e072

Charbonier, J., Malán, R., y Bassani, V. (2017). Introducción a la Agroecología. Colonia, Uruguay: Centro Emmanuel.

Deleuze, G., y Guattari, F. (2008). Mil Mesetas. Capitalismo y Esquizofrenia. Valencia: Pretextos.

Deleuze, G. (2006). Spinoza: filosofía práctica. Buenos Aires: Tusquets Editores.

Deleuze, G. (2003). Deux régimes de fous. Textes et entretiens 1975-1995. Lapoujade, D. Paris: Minuit.

Dirección de Estadísticas Agropecuarias (DIEA - MGAP) (2018). Anuario Estadístico Agropecuario. Recuperado de http://www.mgap.gub.uy/unidad-organizativa/oficina-de-programacion-y-politicas-agropecuarias/publicac iones/anuarios-diea

Duché-García, A., Bernal-Mendoza, H., Ocampo-Fletes, I., Juárez-Ramón, D., y Villarreal-Espino, A. (2017). Agricultura de traspatio y agroecología en el proyecto estratégico de seguridad alimentaria (PESA-FAO) del Estado de Puebla. Agroecologia, Sociedad y Desarrollo, 14(2), 263-281. Recuperado de http://revista-asyd.mx/in dex.php/asyd/article/view/592/188

Fernández, E., y Piñeiro, D. (2008). Organizaciones rurales. En M. Chiappe, M. Carámbula, y E. Fernández (Coord.). El campo uruguayo: una mirada desde la sociología rural (pp. 127-152). Montevideo: Facultad de Agronomía Universidad de la República.

Florit, P. (2011). La cadena forestal celulósica en Uruguay: contexto para la ampliación del Puerto de La Paloma. En D. E. Piñeiro (Coord.), Repercusiones de las inversiones forestales: la ampliación del Puerto de La Paloma (pp. 13-42). Montevideo: Universidad de la República.

Gago, V. (2014). La razón neoliberal. Economías barrocas y pragmática popular.Buenos Aires: Tinta limón.

Gazzano Santos, I., y Gómez Perazzoli, A. (2015). Agroecología en Uruguay. Agroecología, 10(2), 103-113. Universidad de Murcia. Recuperado de https://revistas.um.es/agroecologia/article/view/300871/216291

Gutiérrez, R. (2017). Horizontes comunitario-populares. Producción de lo común más allá de laspolíticas estado-céntricas. Madrid: Traficantes de sueños.

Hecht, S. (1999). La evolución del pensamiento agroecológico. En M. Altieri (Coord.), Agroecología: Bases cientificas para una agricultura sustentable (pp. 15-30). Montevideo: Ed. Nordan-Comunidad.

IFOAM (2008). Sistemas Participativos de Garantia: estudios de caso en América Latina. Alemania: International Federation of Organic Agriculture Movements.

Instituto Nacional de Estadística (INE) (2011). Censo General Agropecuario 2011. Montevideo: MInisterio de Ganadería, Agricultura y Pesca/DIEA. Recuperado de http://www.mgap.gub.uy/sites/default/files/multimedi a/censo2011.pdf

Laclau, E. (2008). Debates y combates. Por un nuevo horizonte de la política. Buenos Aires: Fondo de Cultura Económica.

Laclau, E., y Mouffe, Ch. (2010). Hegemonía y estrategia socialista: hacia una radicalización de la democracia. Buenos Aires: Fondo de Cultura Económica.

Latorre, R. (1991). Los asalariados rurales en el Uruguay. Montevideo: FAgro-UdelaR. 
Ministerio de Ganadería, Agricultura y Pesca (MGAP) (2017). Importaciones de Productos Fitosanitarios 2017. Recuperado de http://www.mgap.gub.uy/noticia/unidad-organizativa/direccion-general-de-servicios-agricolas /07-02-2018/importaciones-de

Ministerio de Ganadería, Agricultura y Pesca (MGAP) (2014). Agricultura familiar en Uruguay. Montevideo: Dirección General de Desarrollo Rural (DGDR). Recuperado de http://www.mgap.gub.uy/sites/default/files/ multimedia/pptpafregistros2015.pdf

Oyhantçabal, G., y Narbondo, I. (2008). Radiografía del agronegocio sojero. Descripción de los principales actores y los impactos socio-económicos en Uruguay. Montevideo: REDES-AT.

Panizza, F. (1990). Uruguay: Batllismo y después. Montevideo: Ediciones de la Banda Oriental.

Pérez Orozco, A. (2014). Subversión feminista de la economía. Madrid: Traficantes de Sueños.

Piñeiro, D. (2014). Asalto a la tierra: el capital financiero descubre el campo uruguayo. En G. Almeyda et al. (coord.). Capitalismo: Tierra y poder en América Latina (1982-2012) (pp. 215-257). Buenos Aires: Ediciones Continente.

Piñeiro, D., y Moraes, M. I. (2008). Los cambios en la sociedad rural durante el siglo XX. En B. Nahum (Coord.). El Uruguay del siglo XX. La Sociedad (pp. 105-136). Departamento de Sociología, Facultad de Ciencias Sociales. Montevideo: Banda Oriental.

Piñeiro, D., y Cardeillac, J. (2018). El Frente Amplio y la política agraria en el Uruguay. En C. Kay y L. VergaraCamus (Comp.), La cuestión agraria y los gobiernos de izquierda en América Latina. Campesinos, agronegocio y neodesarrollismo (pp. 259-286). Buenos Aires: CLACSO.

Real de Azúa, C. (1984).¿¿Uruguay: una sociedad amortiguadora? Montevideo: Ciesu.

Riella, A., y Mascheroni, P. (2019). La organización sindical de los trabajadores agrarios en Uruguay: origen, trayectoria y perspectivas. Mundo Agrario, 20(43), e104. https://doi.org/10.24215/15155994e104

Riella, A., y Romero, J., (2014). Continuidades y rupturas en la estructura agraria en el Uruguay del siglo XXI. Pampa: Revista interuniversitaria de estudios territoriales, 10, 159-172. Recuperado de https://dialnet.unirioja.es/servle $\mathrm{t} /$ articulo? codigo $=4961418$

Roldán Rueda, H. N., Gracia, M. A., y Terán. M. (2018). Los mercados locales alternativos en México y Colombia: resistencias y transformaciones en torno a procesos de certificación. Cuadernos de Desarrollo Rural, 15(82), 1-17. Recuperado de https://revistas.javeriana.edu.co/index.php/desarrolloRural//issue/view/1354

Serra Borsatto, R., y Simoes do Carmo, M. (2012). Agroecologia e sua epistemologia. Interciencia, 37(9), 711-716. Recuperado de https://www.interciencia.net/wp-content/uploads/2018/01/711-1\%C2\%BA-e-BORSATTO -6.pdf

Soler, M., y Pérez, D. (2014). Alimentación, agroecología y feminismo: superando los tres sesgos de la mirada occidental. En E. Siliprandi, y G. Zuluaga (Coords.), Género, agroecología y soberanía alimentaria- Perspectivas ecofeministas (pp. 17-40). Barcelona: Icaria Editorial.

Vittori, F. (2018). Le esperienze italiane di Sistemi partecipativi di garanzia. Sociologia urbana e rurale, 116, 110-129. DOI: https://10.3280/SUR2018-116008

Warmling, D., y Moretti Pires, R. (2017). Sentidos sobre agroecología na produçao, distribuiçao e consumo de alimentos agroecológicos em Florianòpolis, SC, Brasil. Interface, 21(62), 687-698. http://dx.doi.org/10.1590/ $1807-57622016.0385$.

\section{BY-NC-SA}

